\title{
Understanding attentional modulation of binocular rivalry: a framework based on biased competition
}

\author{
Kevin Conrad Dieter ${ }^{1,2}$ * and Duje Tadin ${ }^{1,2,3}$ \\ ${ }^{1}$ Department of Brain and Cognitive Sciences, University of Rochester, Rochester, NY, USA \\ ${ }^{2}$ Center for Visual Science, University of Rochester, Rochester, NY, USA \\ ${ }^{3}$ Department of Ophthalmology, University of Rochester, Rochester, NY, USA
}

\section{Edited by:}

Theofanis Panagiotaropoulos, Max

Planck Institute for Biological

Cybernetics, Germany

\section{Reviewed by:}

Ming Meng, Dartmouth College, USA

Teng Leng Ooi, Pennsylvania College

of Optometry at Salus University,

USA

Urte Roeber, University of Leipzig,

Germany

\section{${ }^{*}$ Correspondence:}

Kevin Conrad Dieter, Department of

Brain and Cognitive Sciences,

University of Rochester, Meliora 358,

RC Box 270268, Rochester, NY

14627-0268, USA.

e-mail: kdieter@bcs.rochester.edu

\begin{abstract}
Starting from early scientific explorations of binocular rivalry, researchers have wondered about the degree to which an observer can exert voluntary attentional control over rivalry dynamics. The answer to this question would not only reveal the extent to which we may determine our own conscious visual experience, but also advance our understanding of the neural mechanisms underlying binocular rivalry. Classic studies, intriguingly, reached contradictory conclusions, ranging from an absence of attentional control, as advocated by Breese, to nearly complete control of rivalry dynamics, as reported by Helmholtz. Recent investigations have revisited this question, but the results have continued to echo the conflicting findings of earlier studies, seemingly precluding a comprehensive understanding of attentional effects on rivalry. Here, we review both classic and modern studies, and propose a unifying framework derived from the biased competition theory of attention. The key assumption of this theory is that the nature of stimulus conflict determines the limits of attentional modulation. For example, a condition in which unresolved stimulus conflict transpires through many levels of visual processing should be very susceptible to attentional control. When applied to binocular rivalry, this framework predicts strong attentional modulations under conditions of unresolved stimulus conflict (e.g., initial selection) and conditions where conflict is resolved at higher levels of visual processing (e.g., stimulus rivalry). Additionally, the efficacy of attentional control over rivalry can be increased by utilization of demanding, behaviorally relevant tasks, and likely through perceptual training paradigms. We show that this framework can help facilitate the understanding and synthesis of a diverse set of results on attentional control over rivalry, and we propose several directions for future research on this interesting topic.
\end{abstract}

Keywords: visual attention, binocular rivalry, bistable perception, biased competition

\section{INTRODUCTION}

Binocular rivalry has long been of fascination to researchers largely because it is a captivating phenomenon that dissociates sensory stimulation from conscious perceptual experience. During binocular rivalry, incompatible images are presented one to each eye, but instead of perceiving a blend of the two images, observers typically report slow, irregular perceptual alternations of the two stimuli (Figure 1). The neural processes underlying the resolution and temporal dynamics of this visual conflict have been the subject of numerous studies and debates over the past century (Blake and Logothetis, 2002; Tong et al., 2006). One issue of particular interest is the influence of selective attention over the dynamics of binocular rivalry. Because binocular rivalry involves an inherent dissociation between sensory input and visual experience, the study of attentional influences over the ebb and flow of perceptual dominances during rivalry holds the allure of shedding light on the extent to which we may determine our own conscious visual experience. Moreover, an understanding of attentional control over binocular rivalry may reveal important insights about the neural mechanisms involved in resolving the conflict that arises during rivalry and, more generally, during other forms of visual competition.

Throughout the history of binocular rivalry research, prominent scientists have reached vastly different conclusions as to the extent to which an observer can voluntarily control perception while viewing incompatible dichoptic patterns. Some concluded that there was a strong degree of voluntary control over binocular rivalry. Notably, Helmholtz (1925) reported that he could completely determine his perception during binocular rivalry while performing a demanding task, such as counting the lines in one of the rival patterns. On the contrary, others believed that no voluntary control over alternations in binocular rivalry was possible (Hering, 1879/1942; Levelt, 1968; Moray, 1970). Breese (1899) reported controlling rivalry in a task similar to that used by Helmholtz, but concluded that the effects were simply the result of eye movements - when he carefully fixated, attentional control diminished. However, Washburn and Gillette (1933) did find a degree of voluntary control over rivalry between afterimages, thereby supporting Helmholtz's assertion that attention can influence rivalry. One of the earliest systematic explorations of this 


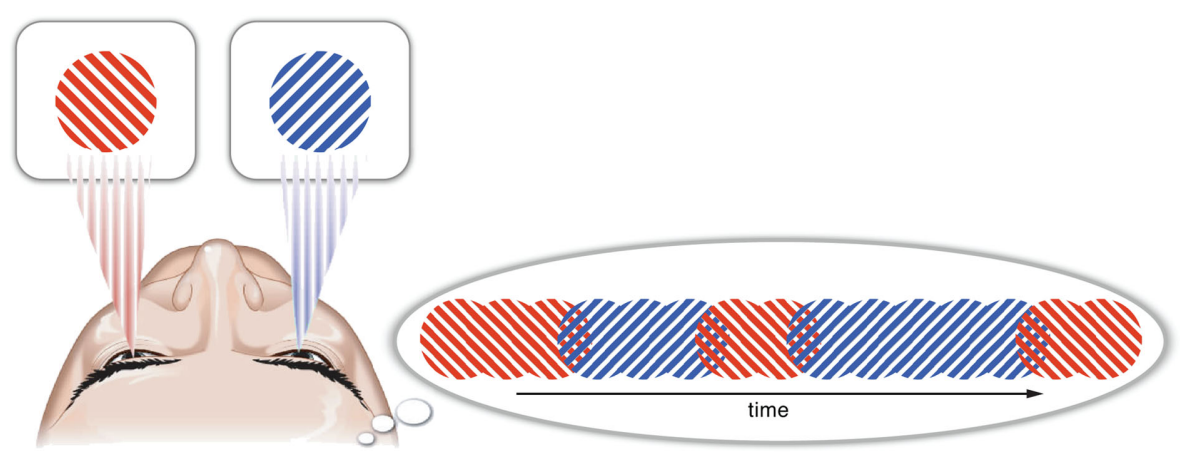

FIGURE 1 | Binocular rivalry occurs when different images are presented, one to each eye. In this situation, observers do not perceive a blend of the two stimuli, but instead experience irregular perceptual alternations between the two images such that only one image is typically perceived at a time. Head image courtesy of Jamie Simon.

research question was by Lack (1978), who found a very strong modulatory effect of voluntary attention over rivalry alternation rates. In Lack's study, participants were asked either to speed the rivalry alternations, or to slow them. This turned out to be an easy task: Lack's subjects were able to increase or decrease the rate of rivalry switches in accordance with their instructions, suggesting a degree of voluntary control over alternations in binocular rivalry.

However, from the onset, a distinction must be made between voluntary control of alternation rates and attentional modulations that are selective to one of the two competing images. The ability to modulate alternation rates during rivalry (Lack, 1978; van Ee et al., 2005) does not necessarily imply selective control over rivalry (Meng and Tong, 2004). In other words, a change in alternation rate can occur without a change in the predominance of one of the two inputs with respect to the other. Furthermore, simple physiological factors can cause changes in the rivalry switch rate. For example, the number of eye blinks is correlated with switch rate (Peckham, 1936), while paralyzing one eye can reduce its dominance during binocular rivalry (McDougall, 1903). These results reveal a simple, and arguably less interesting, link between eye blinks/eye movements and switch rates, and consequently, an easy way to willfully affect the rivalry alternation rate. For these reasons, the present review will mostly focus on studies that have investigated the role of selective attention over rivalry, defined here as cases in which attention boosts predominance of the attended item and/or decreases predominance of the unattended item. However, even in selective control studies, non-attentional factors may influence rivalry dynamics (see Box 1).

The general aim of this review is to propose a unifying attentional framework that can provide an explanation for the wide range of results from studies that investigated the effects of selective attention on rivalry. Although binocular rivalry is a rare perceptual experience, it is fair to assume that rival stimuli are at least in part processed by mechanisms that participate in everyday perception (see Arnold, 2011 for an insightful discussion of this issue). Therefore, we sought an attentional framework developed for visual competition in general that was also able to explain the results obtained during rivalrous viewing. Our general hypothesis, then, is that attentional modulations over binocular rivalry should adhere to the same principles that have been established for visual competition in other, more typical forms. As detailed below, we argue that the biased competition theory of attention (Desimone and Duncan, 1995) can provide an adequate understanding of a seemingly disparate set of findings from studies of rivalry and attention.

\section{A GENERAL FRAMEWORK FOR UNDERSTANDING THE EFFECTS OF SELECTIVE ATTENTION ON RIVALRY}

A fundamental property of binocular rivalry is that it involves sustained visual competition whose outcome fluctuates over time (Figure 1). The processes leading to the dynamic resolution of this conflict and, consequently, to the determination of an observer's visual percept, are thought to reside at multiple levels of the visual hierarchy, with contributions from both low- and high-level mechanisms (Ooi and He, 1999, 2003; Blake and Logothetis, 2002; Tong et al., 2006). Similarly, a key characteristic of attention is that it involves selection among multiple competing alternatives - a process whose outcome results in preferential processing of the “winning” alternative(s) (James, 1890; Broadbent, 1958; Desimone and Duncan, 1995; Egeth and Yantis, 1997; Kastner and Ungerleider, 2000; Reynolds and Chelazzi, 2004; Lavie, 2005). Furthermore, like binocular rivalry, effects of attention occur throughout the visual system (Kastner and Ungerleider, 2000; Treue, 2001; Hochstein and Ahissar, 2002; Serences and Yantis, 2006). These parallels between key properties of rivalry and attention suggest the likely existence of mutual interactions (Leopold and Logothetis, 1999; Stoner et al., 2005). Indeed, as this review shows, a wide variety of attentional effects on rivalry have been documented. However, there is currently no general framework that integrates these empirical results. Here, our aim is to discuss these findings within the theoretical context of a set of rules that have been proposed to govern attentional modulations during typical visual experience; specifically, we apply the principles established by the biased competition theory of attention (Desimone and Duncan, 1995; Desimone, 1998). We find that this framework provides a satisfactory explanation of a range of results. For reasons of simplicity and readability, we do not present a critical evaluation of other theories of attention, but largely take a more focused approach. 


\section{Box 1 | Does attention influence rivalry dynamics by changing effective stimulus contrast?}

Before changes in rivalry dynamics are attributed to attentional mechanisms per se, other factors should be considered. For instance, even a simple increase in the frequency of eye blinks can speed up rivalry (Peckham, 1936). Faster rivalry switching has also been linked to increasing instability of eye fixation (van Dam and van Ee, 2006) and increases in arousal (George, 1936). Experimental control of such factors is particularly important in studies that investigate the effects of attention on the alternation rates in rivalry as they constitute easy ways to either consciously or subconsciously affect rivalry dynamics.

Another important issue is the relationship between attention and changes in effective stimulus contrast (see Paffen and Alais, 2011 for review). Both exogenous and endogenous attention to a stimulus increase its effective contrast (Carrasco, 2006). This is an important issue because changes in stimulus contrast affect rivalry dynamics. For example, increasing the contrast of both rival images will cause them to switch more rapidly, while increasing the contrast of one rival stimulus will decrease dominance durations of the other stimulus (Levelt, 1968). Thus, when one finds an effect of attention on rivalry, that effect may be a direct effect of attention or an indirect effect that is due to attention-dependent changes in stimulus contrast. Indeed, slowing of alternation rates under conditions of diverted attention may be explained by a corresponding decrease in effective stimulus contrast (Paffen et al., 2006; Paffen and Hooge, 2011). Interestingly, the magnitude of the slowing seems to depend on the nature of the stimuli, with higher-level stimuli such as faces and houses showing more slowing with diverted attention than orthogonal gratings (van Ee et al., 2005; Alais et al., 2010a; also see section Effects of Attention on Binocular Rivalry Dynamics). On the other hand, attention-dependent increases in effective stimulus contrast may explain triggering of rivalry alternations caused by exogenous attentional cuing (Paffen and van der Stigchel, 2010). Changes in effective contrast, however, do not explain all effects of diverting attention away from rival stimuli (Pastukhov and Braun, 2007). Indeed, there are several instances where rivalry slowing occurs when attention is directed toward rival stimuli (Chong et al., 2005; van Ee et al., 2009), and additional examples of more complex patterns of results that cannot be easily explained by attention-dependent changes in stimulus contrast (Alais et al., 2010a). Changes in effective stimulus contrast should also be considered in studies that investigated selective attentional control of rivalry, that is, studies where attention was directed to only one of the rival targets (Chong et al., 2005; Hancock and Andrews, 2007; see section Behavioral Relevance Promotes Attentional Control). While both of these studies found an increase in the predominance of the attended item, each was caused by different factors. Chong et al. (2005) found that selective attention boosted the average dominance durations of the attended item, with no change to the unattended item. On the other hand, Hancock and Andrews reported a decrease in the average dominance duration of the unattended item, with no change to the attended item. If attention simply increased the effective stimulus contrast of the attended item throughout the experiment then, in accordance with Levelt's (1968) second proposition, one would expect to see results similar to those reported by Hancock and Andrews. However, in these studies observers attended to the target stimulus only when it was dominant, making it unlikely that its effective contrast was affected while it was suppressed from awareness. Indeed, when a stimulus' contrast is physically increased only during its dominance periods, its dominance durations increase with no changes to the dynamics of the other rival stimulus (Mueller and Blake, 1989; Chong et al., 2005).

In summary, there are a number of indications that attention-dependent changes in effective stimulus contrast may explain some effects of attention on rivalry. This simple explanation, however, does not fully account for all of the results, indicating that attention per se likely has additional effects on rivalry. It will be important for future research to isolate both indirect and direct effects of attention. Importantly, simply demonstrating that attentional modulation effects can be mimicked by changes in physical stimulus contrast is insufficient to conclude that the observed effects of attention are indirect. Such a conclusion will require actual measurement of attention-induced changes in stimulus contrast during rivalry and subsequent testing of whether measured contrast changes are sufficient to replicate the effects of attention on rivalry dynamics.

The fundamental role of attention is to modulate neural processes in order to prioritize attended items. In nearly all visual scenes, there are multiple items that are in competition for neural resources. The biased competition theory of attention (Desimone and Duncan, 1995; Desimone, 1998) argues that the very existence of this competition is central to the understanding of attention. Accordingly, the effects of attention can only be understood in so far as they lead to the resolution of conflict between stimuli - without competition there is no need for attention. For example, attention can easily modulate neural responses to spatially overlapping stimuli (Serences et al., 2004; Liu et al., 2007; Ciaramitaro et al., 2011) as they are in clear competition. Similarly, when two stimuli fall within the same neural receptive field, attention can boost the processing of the attended item relative to the other unattended items (Moran and Desimone, 1985; Reynolds et al., 1999). Importantly this attentional boost is the strongest when the competing stimuli fall inside the neuron's receptive field, as compared to the case when the unattended stimuli are outside the receptive field (Motter, 1993; Luck et al., 1997). Similar results are found in human fMRI studies. When multiple items are presented in a visual scene, attentional modulations of BOLD responses are seen only in visual areas in which the items fall within the same neural receptive fields (i.e., compete within a receptive field), but not in earlier visual areas where receptive fields are too small to "see" more than one item (i.e., competition is across multiple receptive fields; Kastner et al., 1998; Beck and Kastner, 2009). Overall, these results highlight the importance of competitive interactions between stimuli in enabling attentional modulations, and suggest that only unresolved conflict should be subject to attentional modulation. This hypothesis was recently tested by McMains and Kastner (2011), who manipulated perceptual grouping in order to modulate stimulus conflict. Spatially distributed stimuli will compete when they are placed such that multiple stimuli fall within the receptive field of a single neuron; however, perceptual grouping can reduce the magnitude of competitive interactions by integrating multiple stimuli into a single unified whole (Wertheimer, 1938; Tadin et al., 2002). Indeed, when perceptual grouping was strong, attentional modulations of the BOLD signal were much smaller than when the same stimuli formed weak perceptual groups (McMains and Kastner, 2011, 
see Figure 2). This simple study shows that the degree of stimulus conflict determines the magnitude of attentional effects. Taken together, these findings show that unresolved competition in the visual system is important, and likely necessary, to observe selective attentional modulation of neural processing.

These principles have direct implications for understanding attentional modulations of binocular rivalry. By definition, binocular rivalry involves conflict between two spatially corresponding stimuli. According to the biased competition theory of attention, attentional control over rivalry dynamics should depend first on the presence of stimulus conflict, and also on how and when this conflict is resolved. For example, conflict in binocular rivalry remains unresolved for a brief period after the stimuli are initially presented (Wolfe, 1983), which predicts a large degree of attentional control over early rivalry dynamics (see section Effects of Attention on Initial Selection). In contrast, ongoing rivalry contains periods of clear conflict resolution (when perceptual dominance of one stimulus occurs), and, consequently, should be less susceptible to attentional control (see section Effects of Attention on Binocular Rivalry Dynamics). The second assumption of the biased competition theory is that attentional modulations occur within the same neural substrate where stimulus competition takes place. This again has clear implications for binocular rivalry. For example, if the resolution of conflict during rivalry is limited to low-level, bottom-up mechanisms, then there are few opportunities for attentional modulation. Moreover, any attentional modulation should be biased toward the location in the visual system at which the rival stimuli compete (Beck and Kastner, 2009), which in this case should be early visual mechanisms. On the other hand, if conflict resolution (i.e., determination of perceptual dominance) is a high-level process, that suggests the presence of unresolved conflict throughout the visual hierarchy, and consequently more opportunities for attentional modulation. Finally, as an added benefit, applying the framework outlined in this paragraph should help explain not only the effects of attention on rivalry, but also give insight into the mechanisms of binocular rivalry per se. Specifically, determining the degree of attentional control over a certain aspect of binocular rivalry, might give insights into the nature of underlying visual competition (cf., Mitchell et al., 2004).

The straightforward application of this framework, however, is complicated by two important factors. First, as discussed below, rivalry almost certainly does not involve exclusively low- or highlevel processes (Blake and Logothetis, 2002). Second, treating rivalry as a process carried out by a single mechanism is overly simplistic. Instead, binocular rivalry seems to be mediated by an aggregate of related processes that determine different aspects and stages of its dynamics, including the instigation of rivalry, the initial perceptual selection, and subsequent alternations of dominance and suppression (Alais and Blake, 2005). Each of these processes may be differentially susceptible to attentional modulation, precluding a simple conclusion about effects of attention on rivalry. In this review, we will consider these complications as we apply the proposed attentional framework.

\section{EFFECTS OF ATTENTION ON INITIAL SELECTION}

When two stereoscopically compatible images are presented, the visual system almost instantly fuses the two images into a 3D percept (Cumming and DeAngelis, 2001; Blake and Wilson, 2011). However, when two incompatible images are presented, it takes some time for binocular rivalry to begin. Perhaps because of the overwhelming amount of binocularly compatible information that we receive throughout our lives, the visual system first attempts to fuse the inputs from the two eyes by default. For example, orthogonal dichoptic gratings that are presented very briefly will "abnormally fuse," giving rise to a percept of a plaid (Wolfe, 1983; de Belsunce and Sireteanu, 1991). With typical stimuli, exclusive dominance of one of the stimuli occurs only after about
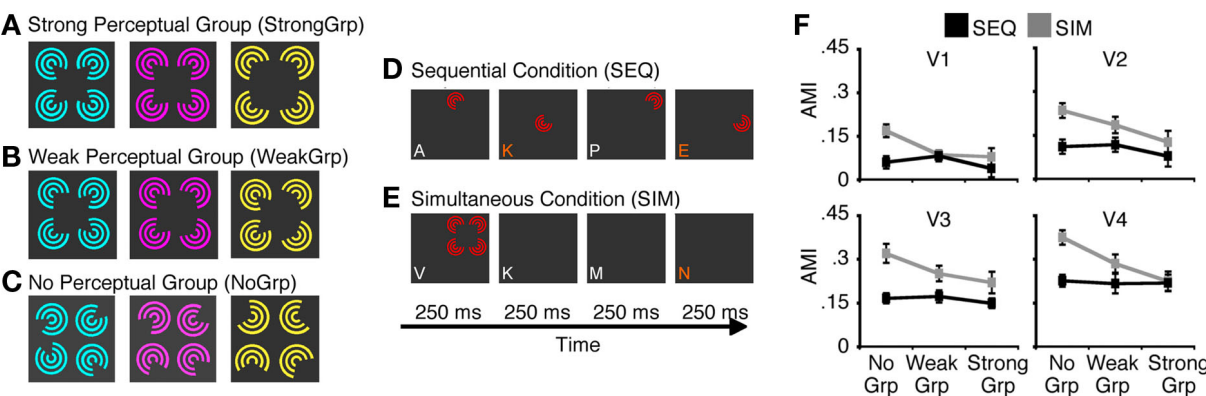

FIGURE 2 | A study by McMains and Kastner (2011) demonstrates that susceptibility to attentional modulation is dependent on the degree of unresolved stimulus conflict. (A-C) Inducer stimuli used in the experiment, which varied in the strength of perceptual grouping, ranging from strong grouping (A) to no perceptual grouping (C). In these displays, stimulus conflict decreases as the strength of perceptual grouping increases. (D,E) Illustrations of experimental conditions and tasks. In the sequential condition (D), inducers are presented in sequence, which precludes competitive interactions among stimuli. In the simultaneous condition (E), all inducers are presented at the same time. This typically results in suppressive interactions among stimuli - a result thought to indicate their competition for neural resources (Kastner et al., 1998; Reynolds et al., 1999). To estimate susceptibility to attentional modulation, observers were asked to perform either a demanding RSVP task at fixation (attention diverted task) or luminance detection task on one of the inducers (attention deployed task). This allowed computation of attentional modulation indices (AMI), which quantified how much responses increased when attention was directed toward the inducer stimuli. (F) The results showed that attentional modulation was strongest in the simultaneous condition and when inducer stimuli did not from a perceptual group. This key finding indicates that the unresolved competition between stimuli is linked with strong susceptibility to attentional modulation. Adopted from McMains and Kastner (2011) with permission from the Society for Neuroscience. 
$150 \mathrm{~ms}$ (the fusion period can be shorter for pairs of stimuli where one is perceptually much stronger, Su et al., 2011). This fusion period indicates the presence of unresolved conflict throughout the visual system, and a possible role for higher-level factors to influence rivalry initiation. For example, imagine a real-world circumstance in which an object is occluded, but the observer stands such that his left eye is able to see the occluded object, while the right eye cannot. In such cases, binocular rivalry does not occur, even though each eye's input is incompatible with the other (Shimojo and Nakayama, 1990; Arnold, 2011). On the other hand, even identical inputs can be made to rival or fuse depending on how they are interpreted in context (Andrews and Lotto, 2004). These results indicate that there is some flexibility in how compatible and incompatible binocular inputs are initially processed, which may result from low-level (i.e., Shimojo and Nakayama, 1990) or higher-level (i.e., Andrews and Lotto, 2004) factors. Importantly, in relation to the framework proposed in this review, this suggests a possible role for attentional modulation during initial selection. Indeed, numerous investigations have shown strong attentional modulations over initial selection in binocular rivalry (Ooi and He, 1999; Mitchell et al., 2004; Chong and Blake, 2006; Hancock and Andrews, 2007; Kamphuisen et al., 2007).

In one such study by Mitchell et al. (2004), object-based exogenous (i.e., involuntary) attention was shown to bias initial dominance in binocular rivalry. The experimental paradigm started with the presentation of two superimposed transparent surfaces to both eyes (Figure 3A). On each trial, one surface was cued by a brief translation - a manipulation designed to exogenously draw attention to the cued surface. After $150 \mathrm{~ms}$, one of the surfaces was removed from each eye, leaving two incompatible surfaces in the two eyes and resulting in the initiation of binocular rivalry. The key result was that the cued grating was about three times more likely to be perceived as dominant during the initial period of binocular rivalry (Figure 3B). This effect of attention was specific to initial dominance, disappearing $2 \mathrm{~s}$ after the presentation of the exogenous cue. Subsequent study by Chong and Blake (2006) reported similar results for endogenous (i.e., voluntary) attention. Their paradigm involved the binocular presentation of two superimposed gratings, with subjects instructed to track either rotation or spatial frequency changes of one of the two gratings. After $5 \mathrm{~s}$, one grating was removed from each eye to initiate binocular rivalry (again, by leaving two incompatible surfaces in two eyes). Results revealed a twofold bias of the initial dominance in favor of the cued stimulus. This effect was only observed on trials where subjects correctly tracked stimulus changes, indicating an important role of sustained endogenous attention.

These studies convincingly demonstrate that both exogenous and endogenous attention can bias initial selection during binocular rivalry. This susceptibility to attentional modulation may be explained by the temporal dynamics that characterize the initial presentation of incompatible binocular stimuli. Here, the delayed onset of exclusive dominance of one of the rival targets provides a period of unresolved conflict between two rival stimuli. As outlined above, such unresolved competition should allow for strong attentional modulations. Moreover, because neither stimulus is perceptually dominant in the first $150 \mathrm{~ms}$, there is unresolved competition throughout the visual hierarchy. Thus, there are a range of

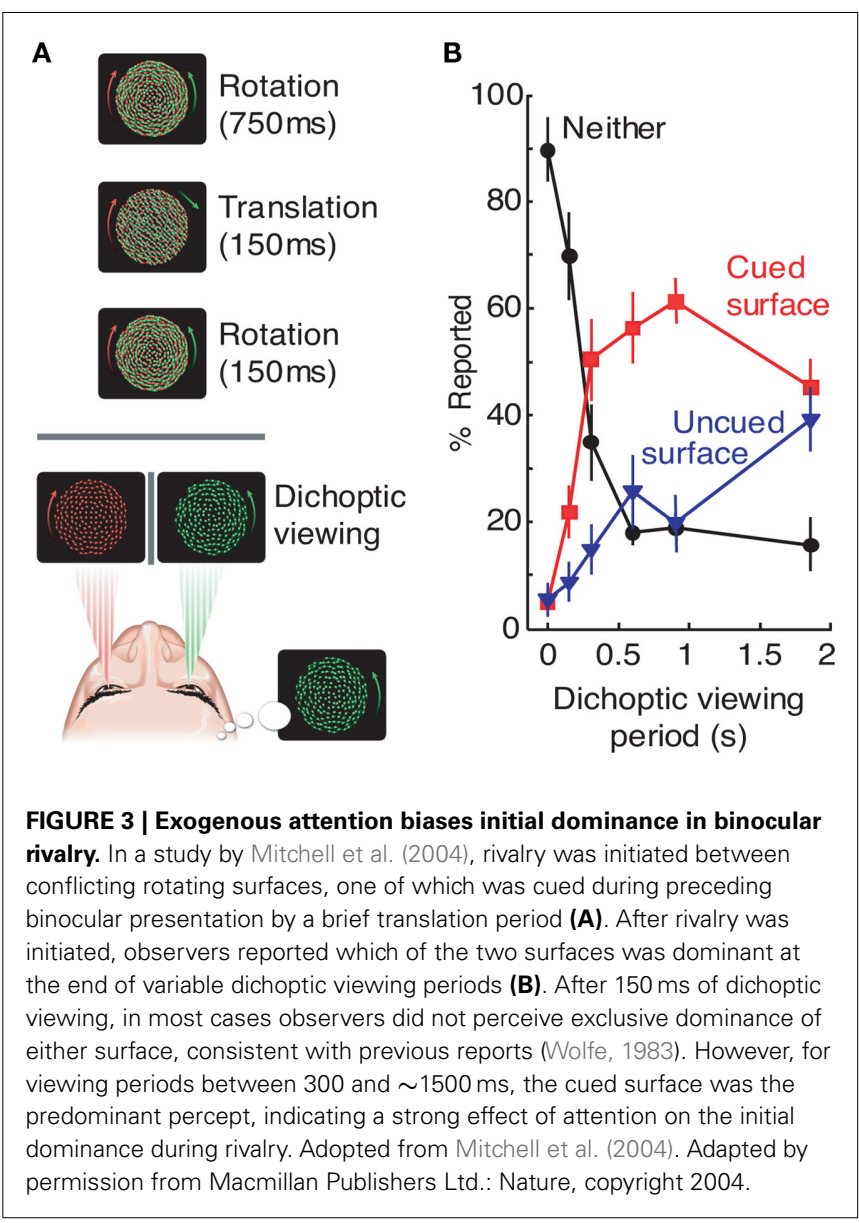

levels at which attentional modulations may occur (Beck and Kastner, 2009). Interestingly, with some stimuli it is possible to resolve interocular competition in as little as $30 \mathrm{~ms}$ (Su et al., 2011). Our proposal is that for such stimuli, the magnitude of attentional control over initial dominance would be much smaller. Additionally, recent EEG results (Zhang et al., 2011) suggest that, in fact, attention may be necessary for the abnormal fusion of two rival stimuli to transition into rivalry alternations (but see Roeber et al., 2011 for a different result; also see Box 2). However, even though this argument may explain why attentional modulation of initial dominance is strong, it also makes it harder to pinpoint the exact neural mechanisms that are involved (see Box $\mathbf{3}$ for future directions that may resolve this issue).

\section{EFFECTS OF ATTENTION ON BINOCULAR RIVALRY DYNAMICS}

Following a brief period of abnormal fusion and subsequent determination of initial dominance in binocular rivalry, rivalry enters its characteristic dynamic of perceptual alternations between the two incompatible stimuli. Because there is considerable evidence that the mechanisms underlying the dynamics of sustained binocular rivalry are at least partially distinct from those underlying initial selection (Wolfe, 1983; de Belsunce and Sireteanu, 1991; Carter and Cavanagh, 2007; Bartels and Logothetis, 2010; Stanley et al., 2011), it is important to determine whether the nature of selective 


\section{Box 2 | Does binocular rivalry require attention?}

The resolution of binocular rivalry requires selection of one rival image for dominance, and another for suppression. Since selection is a fundamental feature of the attentional system, this fact raises the question of whether the processes giving rise to perceptual fluctuations during binocular rivalry actually require that attention be deployed to the rival stimuli. However, getting a clear answer to this question is precluded by a fundamental problem in all behavioral investigations of attention and rivalry. If one wants to investigate how two processes interact, one should first understand each process in isolation, and then examine what happens when the two are combined. However, such a clean design cannot be applied to the behavioral study of attentional effects on binocular rivalry due to the simple fact that reporting a subjective perceptual state during rivalry requires directing attention toward rival stimuli. Therefore, it is impossible to behaviorally measure rivalry dynamics in the absence of attention. Some studies have tried to circumvent this limitation by asking observers to attend to rival stimuli only sporadically, and then using the observers' reports during these brief periods of attending to infer rivalry dynamics during preceding periods of diverted attention (Cavanagh and Holcombe, 2006; He et al., 2007; Pastukhov and Braun, 2007). The results suggest that rivalry either considerably slows down or possibly stops alternating outside of the scope of attention. However, because attention must be periodically directed to the rival stimuli (in order for observers to make responses), these studies do more to highlight the impossible task of behaviorally measuring rivalry dynamics without attention than to actually circumvent this limitation. This fundamental problem, fortunately, can be avoided by using non-behavioral methods of assessing rivalry dynamics. Specifically, neuroimaging methods can be used to characterize neural signature(s) of rivalry alternations, which then can be examined with and without attention. This was done in a recent EEG study by Zhang et al. (2011), who found that when attention was directed away from the rival stimuli, rivalry stopped. These intriguing findings suggest that attention may be necessary for the resolution of conflict in binocular rivalry. However, an opposite finding was reported in an event-related potential (ERP) study by Roeber et al. (2011). Evidently, a simple answer to this important question remains elusive.

attentional modulation might also differ. The first modern study to address this question was by Meng and Tong, who instructed subjects to hold one of the two rival stimuli dominant for as long as possible. Consistent with the older hypothesis by Moray (1970), the results showed essentially no effect of attention (Figure 4). There were no changes in the average dominance durations or total predominance in favor of the held item or against the ignored stimulus. Evidently, simply directing endogenous attention toward one of the rival stimuli has little effect over the dynamics of binocular rivalry (as discussed in the following section, different results are found if attended stimuli are behaviorally relevant). In contrast, when the same subjects were asked to perform an analogous task with the bistable Necker cube, strong attentional modulation was observed (Figure 4; Meng and Tong, 2004; also see Toppino, 2003). Similar results are found for other types of ambiguous visual stimuli, including apparent motion (Suzuki and Peterson, 2000) and 3D structure-from-motion (Hol et al., 2003). In fact, voluntary attention to the alternate perceptual explanation is often required see ambiguous figure reversals (personal in-class observation with E. G. Boring's Young girl/Mother-in-law image). One explanation of these results is that the nature of competition in ambiguous stimuli is high-level, and therefore it is easily affected by attentional control. Overall, this contrast between rivalry and ambiguous figures supports the hypothesis that conflict during binocular rivalry is resolved at early stages of visual processing in a bottom-up fashion (Blake, 1989), thereby limiting the degree of attentional modulation (McMains and Kastner, 2011).

Competition during binocular rivalry, however, is not exclusively confined to early, monocular mechanisms, but may involve different levels of the visual system depending on the nature of the competition (Blake and Logothetis, 2002; Lee, 2004). One notable case that reveals an important role of higher-level processes is stimulus rivalry (Logothetis et al., 1996). In this paradigm, each eye receives conflicting input, but the ocular configuration is rapidly swapped between the eyes $(\sim 3 \mathrm{~Hz})$. Under these specific conditions rivalry dynamics remain largely unchanged (Logothetis et al., 1996; Lee and Blake, 1999), precluding an eye-based explanation.

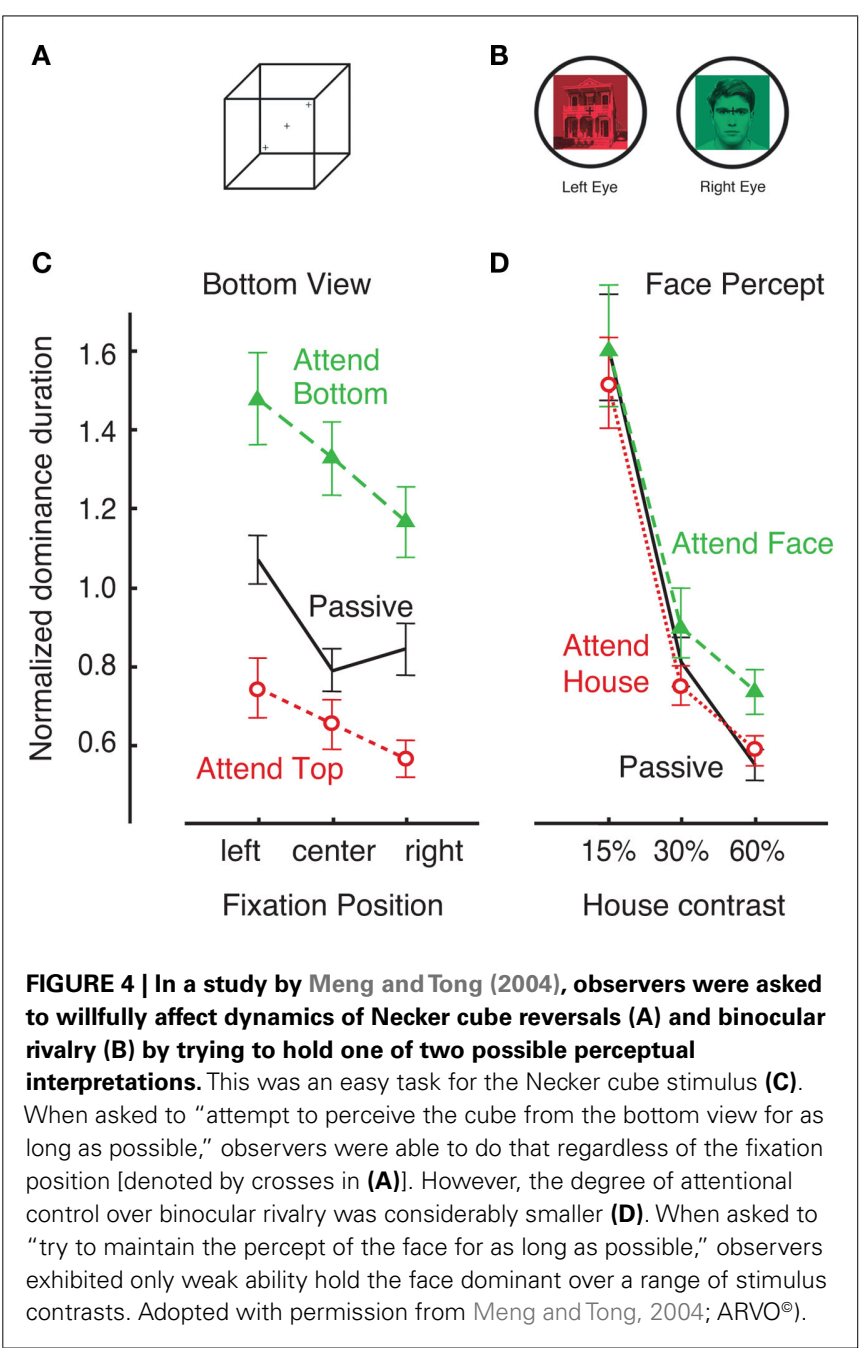

Importantly, visual competition leading to these dynamics by definition involves a conflict between two stimuli rather than a conflict 
between two eyes and likely involves higher visual areas (Pearson et al., 2007). Modeling studies indicate that stimulus rivalry occurs for stimuli that bypass the low-level competition that normally occurs at early, monocular stages (Wilson, 2003; Freeman, 2005; Tong et al., 2006). Another example involves composite stimuli created by fragmenting two larger images and pseudorandomly assigning the corresponding fragments to two eyes (i.e., each eye would see a mosaic containing complementing components of two source images). For such stimuli, rather than perceiving rivalry between two mosaic images, subjects often perceive the unfragmented source images (Diaz-Caneja, 1928, translated by Alais et al., 2000; Kovács et al., 1996). In addition, globally grouped motion (Alais and Blake, 1998), surface contours (Ooi and He, 2003; van Bogaert et al., 2008), textures with common luminance or color (Silver and Logothetis, 2004), as well as other grouped stimuli (Logothetis, 1998) can influence locally competing rival stimuli. These effects indicate involvement of object-based processes operating at multiple stages throughout the visual hierarchy. Because of the higher-level nature of visual competition in these examples, the framework introduced in this review predicts a greater degree of attentional control. While this specific hypothesis remains to be tested, there are some indications that these forms of visual rivalry are more susceptible to top-down factors. For example, unlike eye rivalry, stimulus rivalry requires high levels of pattern coherence, indicating a key role of object-based mechanisms (Bonneh et al., 2001). An analogous dissociation is evident in the perceptual stabilization that occurs when rival stimuli are presented intermittently (Leopold et al., 2002). Object features, such as color, are the primary factor determining perceptual stabilization during intermittent stimulus rivalry (Pearson and Clifford, 2004). On the other hand, low-level eye of origin is almost the sole determinant of perception during intermittent binocular rivalry (Chen and He, 2004; Pearson and Clifford, 2004). Additional evidence that the level where stimuli compete affects their susceptibility to attentional modulation comes from studies that investigated the slowing of rivalry that occurs when attention is diverted (see Box 1). Observed effects are larger for rivalrous face/house stimuli than for orthogonal gratings (van Ee et al., 2005; Alais et al., 2010a). This is likely due to the fact that conflict between faces and houses arises later in the visual hierarchy than does conflict between gratings (cf., Beck and Kastner, 2009).

Arguably the strongest indication that stimulus rivalry may be more susceptible to attentional modulation comes from a recent study by Silver and Logothetis (2007). Here, one of two dichoptically presented orthogonal gratings was embedded with a conspicuous visual tag. Throughout the course of a trial, this tag either remained with the same stimulus (and switched eye at every eye swap) or remained in the same eye (and switched stimulus on each eye swap). When the tag remained in one eye, subjects were biased toward perceiving fast stimulus switches, indicating sustained periods of eye dominance. Importantly, when one of the two stimuli was tagged, subjects tended to experience slow, irregular stimulus alternations (i.e., they perceived stimulus rivalry). In this study, participants likely used attentional tracking strategies to follow the cue. During conditions where the tag remained with one stimulus, this effectively deployed selective attention to that tagged stimulus. Although the goal of this study was not to study attentional modulations, it does suggest stronger attentional control over stimulus rivalry than binocular rivalry. Evidently, as the visual competition is biased toward higher-level mechanisms, the susceptibility to attentional modulation increases.

\section{BEHAVIORAL RELEVANCE PROMOTES ATTENTIONAL CONTROL}

The predominantly low-level nature of conflict during binocular rivalry, however, should not by itself preclude attentional modulations. Although attentional modulations are more closely associated with higher visual processes, attentional effects in early visual areas, including V1 and LGN, are well documented (Treue, 2001; O'Connor et al., 2002; Roberts et al., 2007; Fischer and Whitney, 2009). So, what then may be a reason for weak attentional modulation of conventional binocular rivalry? One reason binocular rivalry continues to fascinate is that it is not a common perceptual experience. This also indicates that we are rarely in situations where we have to select between conflicting inputs arising from two eyes. On the other hand, we frequently select between higher-level stimulus features, such as objects. This ecological difference might explain the resistance of binocular rivalry to attentional control. But, what if one eye's stimulus is behaviorally relevant, for instance through an eye-specific, attentionally demanding task? Would that promote attentional control? Evidence supporting the load theory of attention indicates that the efficacy of attentional deployment critically depends on the effort involved in processing of the attended target, with high loads yielding stronger attentional modulations (Lavie, 2005). Another key question is whether continuing behavioral relevance of one eye's stimulus gradually strengthens any modulatory effects of attention. While we do not normally select between two eyes, the visual system does have the capability to do so. A striking example is amblyopia, a condition where the brain essentially ignores poor visual input from one eye in favor of behaviorally relevant input from the other eye (Campos, 1995; Simons, 2005; Levi and Li, 2009). In a way, amblyopia can be thought of as a limiting case of binocular rivalry, where both eyes are sending differing inputs to the brain, but the visual system learns to ignore the information from the weak eye, thereby resulting in a profound and possibly complete dominance of the stronger eye.

Although amblyopia is clearly a special case and may not involve attentional mechanisms, it demonstrates that an effort to maintain the most relevant visual information may alter the relative dominance of two monocular images. Indeed, recent studies with typical observers have shown that relative dominance can be biased in favor of an attended stimulus if an appropriate attentionally demanding task is used. In one study that addressed this question, participants were instructed to identify small aspect ratio changes in a bullseye pattern presented to one eye, while completing no task when the other eye's stimulus was dominant (Figure 5A; Chong et al., 2005). The results revealed an approximately $50 \%$ increase in the dominance durations of the attended stimulus (Figure 5B). Importantly, for this attentional effect to occur, attention needed to be directed toward the features of one of two rival stimuli - simply performing a demanding task at the same spatial location during dominance periods of the target stimulus was insufficient to bias rivalry dynamics. In a related study, one of two 

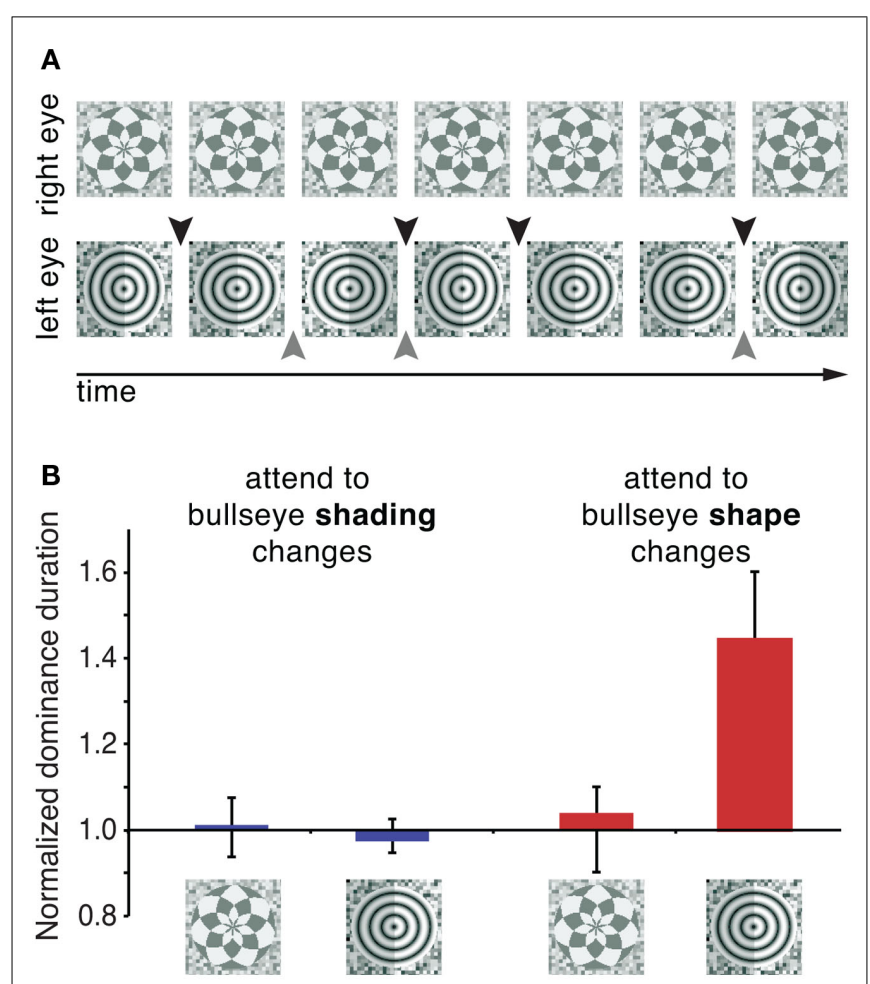

FIGURE 5 | Performing a demanding attentional task promotes selective attentional control over rivalry dynamics. (A) Stimuli used by Chong et al. (2005). One eye views a control stimulus (a radial checkerboard), while the other eye is shown a bullseye pattern. In two separate attentional tasks, observers were instructed to track either shape changes (black arrows) or shading changes (gray arrows) of the bullseye pattern. These tasks were demanding and required sustained attention. For illustration purposes, the magnitudes of the shape and shading changes are greatly exaggerated. Results $(\mathbf{B})$ indicate that completing a demanding task that directs attention toward a rival stimulus lengthens its average dominance duration. On the other hand, an equally demanding task where attention was directed to the same location in space but not to rival stimulus per se did not affect rivalry dynamics. Adopted with permission from Chong et al., 2005; $\mathrm{ARVO}^{\odot}$ ).

orthogonal dichoptic gratings was cued, and participants were instructed to identify small rotations of the cued grating (Hancock and Andrews, 2007). This task also resulted in an increase in the predominance of the attended stimulus. (Interestingly, these two studies measured opposite factors giving rise to this outcome. See Box 1 for more details). These findings indicate that behavioral relevance of a rival stimulus facilitates its attentional susceptibility, a process that likely involves attentional modulation of early visual processes. This argument is supported by recent ERP results indicating that neural changes associated with a demanding attentional task occur at earlier stages when the attended stimulus is engaged in binocular rivalry (Khoe et al., 2008; Mishra and Hillyard, 2009). Specifically, the P1 component, an early ERP component that is believed to reflect extrastriate neural activity, was modulated by attention only when the attended stimulus was presented under dichoptic conditions and rivaled with the unattended stimulus, and not during monocular presentation of the same stimuli. However, even with the utilization of demanding attentional tasks, the magnitude of attentional modulation in these studies (Chong et al., 2005; Hancock and Andrews, 2007) was considerably smaller than attentional effects on initial dominance (see above), indicating that initial selection is more susceptible to attentional control than sustained binocular rivalry.

The use of demanding attentional tasks, however, is not the only way to increase behavioral relevance of a rival target. Other paradigms that increase the relative importance of a rival stimulus also increase its predominance. For example, faces with emotional content dominate over neutral faces during binocular rivalry (Alpers and Pauli, 2006; Bannerman et al., 2008) and emerge faster from perceptual suppression (Yang et al., 2007). Neutral faces that are paired with negative gossip predominate over faces paired with neutral statements (Anderson et al., 2011). In addition, observers' ability to control the alternation rate during rivalry is greatly enhanced if a rival stimulus is paired with a congruent auditory stimulus (van Ee et al., 2009). Even implicitly learned stimulus usefulness biases initial selection in binocular rivalry (Chopin and Mamassian, 2010). In summary, different ways of introducing behavioral relevance (i.e., without using explicit attentional tasks) are very effective at modulating rivalry dynamics. One hypothesis is that at least some of these manipulations work because they enable more effective direction of attention to a rival stimulus a conclusion consistent with the load theory of attention (Lavie, 2005).

\section{PLASTICITY OF ATTENTIONAL EFFECTS}

Evidence that an eye-specific behavioral task can boost the predominance of the task-relevant stimulus raises two interesting questions. First, increased predominance of the attended stimulus could be as a result of either an attentional boost to the high-level stimulus representation and/or an attentional modulation of low-level monocular processes. Second, the link between behavioral relevance and attentional control raises the question of whether the magnitude of the observed effects could be increased. Perceptual training studies might answer both of these questions. Recent research has revealed that plasticity indeed occurs during prolonged viewing of binocular rivalry (Suzuki and Grabowecky, 2007; Klink et al., 2010), in turn opening a possibility that prolonged attentional control over rivalry may alter its own effectiveness. Moreover, any permanent change in the effectiveness of attentional control allows subsequent determination of whether observed changes are specific to the trained eye and/or the trained stimulus.

An early series of studies by Lack indicated that voluntary control over rivalry alternation rate may be subject to training. Lack's observers were asked to view rival stimuli and to either speed up or slow down their alteration rates - a task that is relatively easy to accomplish (Lack, 1978; van Ee et al., 2005). Interestingly, over the course of 10 days, observers became considerably better at controlling their own switch rates (Lack, 1978). As discussed above (also see Box 1), non-attentional factors may explain such changes in alternation rates. Still, Lack's results warrant a more controlled investigation into the plasticity of attentional control during rivalry. More recent work (Suzuki and Grabowecky, 2007) revealed that long-term observation of binocular rivalry indeed modulates alternation rates. However, observers in this study 


\section{Box 3 | Questions for future research.}

- The attentional framework outlined in this review can help synthesize results from a number of behavioral studies that examined the effects of attention on binocular rivalry. However, in most cases, it does not specify where these attentional effects are occurring in the brain (one exception would be eye-specific effects of attentional training). The key complication is that binocular rivalry is a complex mechanism that involves processes spreading out throughout the visual hierarchy (Blake and Logothetis, 2002; Tong et al., 2006). The uncertainties of where exactly rivalry competition takes place carry over to the uncertainty in where behaviorally measured attentional effects occur. Brain imaging, however, holds promise to provide some specificity as to where attentional modulation of rivalry occurs, and, in turn, to help us understand the brain mechanisms involved in rivalry competition. The simple hypothesis motivated by attentional studies (Luck et al., 1997; Beck and Kastner, 2009; McMains and Kastner, 2011) is that the biggest effects of attention should be seen at the stages where rival stimuli are first in competition and not before. It is also possible that some brain areas may play a special role in attentional modulation of rivalry. One such region is the parietal cortex, which is involved in both top-down attention (Behrmann et al., 2004) and has been linked with binocular rivalry (Lumer et al., 1998; Britz et al., 2010; Kanai et al., 2010; Zaretskaya et al., 2010; but see Knapen et al., 2011).

- While biased competition can provide a post hoc explanation of published results on attentional modulation of binocular rivalry, direct empirical tests of this framework are needed. The general hypothesis is that the degree of attentional modulation of rivalry will depend on the unresolved competition between rival stimuli. One specific prediction is that attentional modulation over rivalry dynamics will vary over the course of a single dominance epoch, being weaker at the beginning and stronger near the end. This prediction derives from recent results (Alais et al., 2010b) that show that suppression depth decreases over the course of a dominance period. As strong suppression indicates relatively resolved visual competition, the effects of attentional deployment should vary accordingly.

- However, even after localization of brain area(s) where attentional modulation of rivalry occurs, the exact mechanisms of attentional control over rivalry might still remain a mystery. While physiological changes such as increased alertness and attention-dependent changes in effective stimulus contrast almost certainly play a role (see Box 1), these effects likely do not fully account for attentional modulations of binocular rivalry, particularly under conditions of behavioral relevance of one of the two stimuli.

- An important goal for future research will be to determine the limits of attentional control over rivalry. Was Helmholtz actually correct, after all, about the possibility of complete control over rivalry? Currently, demonstration of complete voluntary control seems elusive, except perhaps after 25 years of intensive meditation training (Carter et al., 2005). Understanding of this limitation will shed light on the conditions under which we may determine our own conscious visual experience.

- If the degree of unresolved stimulus conflict indeed determines susceptibility to attentional modulation, then does an observer's ability to exert attentional control over rivalry fluctuate during different periods of rivalry dynamics? For example, are mixed periods - rivalry periods where an observer perceives a mixture of two stimuli - more susceptible to attentional modulation? If so, could targeted deployment of attention during mixed periods be an effective strategy to significantly prolong predominance of a rival stimulus?

- Although this review focused on modulatory effects of attention on rivalry, a recent study (Zhang et al., 2011) indicates that attention may also have a fundamental enabling role in the initiation of rivalry alternations. However, Roeber et al. (2011) found signatures of rivalry even when attention was diverted. These recent findings open an exciting new line of binocular rivalry research.

- Finally, it will be important to explore possible practical benefits of attentional control over rivalry, particularly its plasticity. Xu et al. (2010) recently demonstrated that perceptual training that combines binocular rivalry and an attentional task can result in improvements of stereopsis. It remains to be determined whether similar paradigms may be used as a behavioral treatment for amblyopia.

passively viewed rival stimuli, so it remains unclear how these changes might be altered by the addition of attentional influences.

Additional suggestions that attentional modulation of rivalry is subject to plasticity come from two recent studies. In a study by Paffen et al. (2008), observers were trained on a directionspecific speed discrimination task over the course of 5 days. After training, the task-relevant (trained) direction and task-irrelevant (ignored) motion direction were pitted against one another in binocular rivalry. Perceptual learning resulted in a decrease in the predominance of the task-irrelevant motion. Furthermore, initial dominance was biased in favor of the task-relevant motion direction. Evidently, prolonged training with a specific stimulus changes its predominance during binocular rivalry. Another study examined whether the pairing of exogenous attention and binocular rivalry could alter sensory eye dominance (Xu et al., 2010; also see Xu et al., 2011a,b). Sensory eye dominance describes a condition, akin to handedness, in which the input from one eye is stronger than that from the other eye, resulting in increased dominance of the stronger eye. In this study, an exogenous attentional cue to the weak eye was followed by a brief presentation of rival gratings. This "push-pull" training led to a significant decrease in the magnitude of sensory eye dominance. The training effects only minimally transferred to other retinal locations and other stimuli, indicating high specificity. Importantly, no changes were found in push only training where no rival stimulus was shown to the stronger eye, suggesting that the involvement of inhibitory interactions during rivalry was key to obtain this low-level plasticity. Preliminary results from our lab (Dieter et al., 2010) show that prolonged training in which observers perform an attentionally demanding task on one rival stimulus (same task as in Chong et al., 2005; see Figure 5A) can lead to increasing voluntary control of rivalry dynamics. The changes giving rise to this additional control were partially eye-specific, as indicated by transfer to untrained stimuli presented to the trained eye.

While more research is needed to understand the plasticity of attentional control over rivalry, it is becoming clear that the limits of attentional effects can be changed through appropriate perceptual training. One striking example is that Tibetan Buddhist monks with over 25 years of practice at "one-point" meditation were able to almost completely control their perception during rivalry (Carter et al., 2005), even though they had no prior experience with binocular rivalry. This unique observation indicates a possibility that complete control over binocular rivalry may be possible with extensive training. 


\section{SUMMARY}

The influence of selective attention over the dynamics of binocular rivalry has been of interest to researchers for over a century. Early investigations came to vastly different conclusions foreshadowing the seemingly diverse set of findings seen in modern studies. The aim of this review is to introduce an attentional framework that can help facilitate the understanding and synthesis of these results. Perhaps reassuringly, the effects of attentional control over binocular rivalry seem to mimic those seen in other paradigms of attentional modulation. Namely, the degree of attentional modulation over rivalry dynamics seems to depend on the presence of stimulus conflict, and the level of the visual system at which conflicting stimuli first compete. This framework predicts strong attentional control under conditions of unresolved stimulus conflict (e.g.,

\section{REFERENCES}

Alais, D., and Blake, R. (1998). Interactions between global motion and local binocular rivalry. Vision Res. 38, 637-644.

Alais, D., and Blake, R. (ed.). (2005). Binocular Rivalry. Cambridge: MIT Press.

Alais, D., O'Shea, R., Mesana-Alais, C., and Wilson, I. (2000). On binocular alternation. Perception 29, 1437-1445.

Alais, D., van Boxtel, J. J., Parker, A., and van Ee, R. (2010a). Attending to auditory signals slows visual alternations in binocular rivalry. Vision Res. 50, 929-935.

Alais, D., Cass, J., O'Shea, R. P., and Blake, R. (2010b). Visual sensitivity underlying changes in visual consciousness. Curr. Biol. 20, 1362-1367.

Alpers, G. W., and Pauli, P. (2006). Emotional pictures predominate in binocular rivalry. Cogn. Emot. 20, 596-607.

Anderson, E., Siegel, E. H., BlissMoreau, E., and Barrett, L. F. (2011). The visual impact of gossip. Science 332, 1446-1448.

Andrews, T., and Lotto, R. (2004). Fusion and rivalry are dependent on the perceptual meaning of visual stimuli. Curr. Biol. 14, 418-423.

Arnold, D. H. (2011). Why is binocular rivalry uncommon? Discrepant monocular images in the real world. Front. Hum. Neurosci. 5:116. doi:10.3389/fnhum.2011.00116

Bannerman, R. L., Milders, M., De Gelder, B., and Sahraie, A. (2008). Influence of emotional facial expressions on binocular rivalry. Ophthalmic Physiol. Opt. 28, 317-326.

Bartels, A., and Logothetis, N. K. (2010). Binocular rivalry: a time dependence of eye and stimulus contributions. J. Vis. 10, 3.

Beck, D. M., and Kastner, S. (2009). Topdown and bottom-up mechanisms in biasing competition in the human brain. Vision Res. 49, 1154-1165.

Behrmann, M., Geng, J. J., and Shomstein, S. (2004). Parietal cortex and attention. Curr. Opin. Neurobiol. 14, 212-217.

Blake, R. (1989). A neural theory of 145-167.

Blake, R., and Logothetis, N. (2002). Visual competition. Nat. Rev. Neurosci. 3, 13-23.

Blake, R., and Wilson, H. (2011). Binocular vision. Vision Res. 51, 754-770.

Bonneh, Y., Sagi, D., and Karni, A. (2001). A transition between eye and object rivalry determined by stimulus coherence. Vision Res. 41, 981-989.

Breese, B. B. (1899). On inhibition. Psychol. Monogr. 3, 1-65.

Britz, J., Pitts, M. A., and Michel, C. M. (2010). Right parietal brain activity precedes perceptual alternation during binocular rivalry. Hum. Brain Mapp. 32, 1432-1442.

Broadbent, D. E. (1958). Perception and Communication. London: Pergamon. Ophthalmol. 40, 23-39.

Carrasco, M. (2006). Covert attention chophysical, neurophysiological and neuroimaging studies. Prog. Brain Res. 154, 33-70.

Carter, O., and Cavanagh, P. (2007). Onset rivalry: brief presentation isolates an early independent phase of perceptual competition. PLoS ONE 2, e343. doi:10.1371/journal.pone. 0000343

Carter, O. L., Presti, D. E., Callistemon, C., Ungerer, Y., Liu, G. B., and Pettigrew, J. D. (2005). Meditation alters perceptual rivalry in Tibetan Buddhist monks. Curr. Biol. 15, R412-R413. binocular rivalry. Psychol. Rev. 96,

Campos, E. (1995). Amblyopia. Surv. increases contrast sensitivity: psy-

initial selection) and conditions where conflict is resolved at higher levels of processing (e.g., stimulus rivalry). In addition, limits on attentional control can be alleviated by the utilization of demanding, behaviorally relevant tasks, and likely through perceptual training paradigms. Future research on this topic (Box 3) will likely reveal not only the extent to which an observer may voluntarily control his or her own perceptual experience, but also new insights into the mechanisms that resolve conflict during binocular rivalry.

\section{ACKNOWLEDGMENTS}

We thank Joel Pearson and Molly Olivia Tadin for manuscript comments. This work was partially supported by Awards P30 EY001319 and T32 EY007125 from the NIH NEI.

Cavanagh, P., and Holcombe, A. O. (2006). Successive rivalry does not occur without attention. J. Vis. 6, 818. doi: $10.1167 / 6.6 .818$

Chen, X., and He, S. (2004). Local factors determine the stabilization of monocular ambiguous and binocular rivalry stimuli. Curr. Biol. 14, 1013-1017.

Chong, S., and Blake, R. (2006). Exogenous attention and endogenous attention influence initial dominance in binocular rivalry. Vision Res. 46, 1794-1803.

Chong, S., Tadin, D., and Blake, R. (2005). Endogenous attention prolongs dominance durations in binocular rivalry. J. Vis. 5, 1004-1012.

Chopin, A., and Mamassian, P. (2010) Task usefulness affects perception of rivalrous images. Psychol. Sci. 21, 1886-1893.

Ciaramitaro, V. M., Mitchell, J. F., Stoner, G. R., Reynolds, J. H., and Boynton, G. M. (2011). Object-based attention to one of two superimposed surfaces alters responses in human early visual cortex. J. Neurophysiol. 105, 1258-1265.

Cumming, B. G., and DeAngelis, G. C. (2001). The physiology of stereopsis. Annu. Rev. Neurosci. 24, 203-238.

de Belsunce, S., and Sireteanu, R. (1991). The time course of interocular suppression in normal and amblyopic subjects. Invest. Ophthalmol. Vis. Sci. 32, 2645-2652.

Desimone, R. (1998). Visual attention mediated by biased competition in extrastriate visual cortex. Philos. Trans. R. Soc. Lond. B Biol. Sci. 353, 1245-1255.

Desimone, R., and Duncan, J. (1995). Neural mechanisms of selective visual attention. Annu. Rev. Neurosci. 18, 193-222.

Diaz-Caneja, E. (1928). On binocular alternation. Ann. Ocul. 721-731.
Dieter, K. C., Melnick, M. D., and Tadin, D. (2010). Eye-specific plasticity induced by binocular rivalry training. J. Vis. 10, 19. doi: 10.1167/10.15.19

Egeth, H., and Yantis, S. (1997). Visual attention: control, representation and time course. Annu. Rev. Psychol. 48, 269-297.

Fischer, J., and Whitney, D. (2009). Attention narrows position tuning of population responses in V1. Curr. Biol. 19, 1356-1361.

Freeman, A. (2005). Multistage model for binocular rivalry. J. Neurophysiol. 94, 4412-4420.

George, R. W. (1936). The significance of the fluctuations experienced in observing ambiguous figures and in binocular rivalry. J. Gen. Psychol. 15, 39-61.

Hancock, S., and Andrews, T. J. (2007). The role of voluntary and involuntary attention in selecting perceptual dominance during binocular rivalry. Perception 36, 288-298.

He, S., Jiang, Y., and Chen, X. (2007). Stabilizing bistable visual patterns through interocular suppression, crowding, and inattention. J. Vis. 7, 362. doi:10.1167/7.9.362

Helmholtz, H. von. (1925). Treatise on Physiological Optics, Vol. 3, trans. J. P. C. Southall. New York: Dover.

Hering, E. (1879/1942). "Spatial sense and movements of the eye," in Handbuch der Physiologie, ed. L. Hermann (Leipzig: Vogel), 3.

Hochstein, S., and Ahissar, M. (2002). View from the top: hierarchies and reverse hierarchies in the visual system. Neuron 36, 791-804.

Hol, K., Koene, A., and van Ee, R. (2003). Attention-biased multi-stable surface perception in three-dimensional structure-from-motion. J. Vis. 3, 486-498. 
James, W. (1890). The Principles of Psychology. New York: Henry Holt.

Kamphuisen, A. P., van Wezel, R. J. A., and van Ee, R. (2007). Interocular transfer of stimulus cueing in dominance selection at the onset of binocular rivalry. Vision Res. 47 , 1142-1144.

Kanai, R., Bahrami, B., and Rees, G. (2010). Human parietal cortex structure predicts individual differences in perceptual rivalry. Curr. Biol. 20, 1626-1630.

Kastner, S., De Weerd, P., Desimone, R., and Ungerleider, L. G. (1998). Mechanisms of directed attention in the human extrastriate cortex as revealed by functional MRI. Science $282,108-111$.

Kastner, S., and Ungerleider, L. G. (2000). Mechanisms of visual attention in the human cortex. Annu. Rev. Neurosci. 23, 315-341.

Khoe, W., Mitchell, J. F., Reynolds, J. H., and Hillyard, S. A. (2008). ERP evidence that surface-based attention biases interocular competition during rivalry. J. Vis. 8, 18.1-18.11.

Klink, P. C., Brascamp, J. W., Blake, R., and Van Wezel, R. J. A. (2010). Experience-driven plasticity in binocular vision. Curr. Biol. 20, 1464-1469.

Knapen, T., Brascamp, J., Pearson, J., van Ee, R, and Blake R. (2011). The role of frontal and parietal areas in bistable perception. J. Neurosci. 31, 10293-10301.

Kovács, I., Papathomas, T. V., Yang, M., and Fehér, A. (1996). When the brain changes its mind: interocular grouping during binocular rivalry. Proc. Natl. Acad. Sci. U.S.A. 93, 15508-15511.

Lack, L. C. (1978). Selective Attention and the Control of Binocular Rivalry. Paris: Mouton.

Lavie, N. (2005). Distracted and confused?: selective attention under load. Trends Cogn. Sci. (Regul. Ed.) 9, 75-82.

Lee, S. H. (2004). Binocular battles on multiple fronts. Trends Cogn. Sci. (Regul. Ed.) 8, 148-151.

Lee, S. H., and Blake, R. (1999). Rival ideas about binocular rivalry. Vision Res. 39, 1447-1454.

Leopold, D., Wilke, M., Maier, A., and Logothetis, N. (2002). Stable perception of visually ambiguous patterns. Nat. Neurosci. 5, 605-609.

Leopold, D. A., and Logothetis, N. K. (1999). Multistable phenomena: changing views in perception. Trends Cogn. Sci. (Regul. Ed.) 3, 254-264.

Levelt, W. J. M. (1968). On Binocular Rivalry. Paris: Mouton.

Levi, D. M., and Li, R. W. (2009). Improving the performance of the amblyopic visual system. Philos. Trans. R. Soc. Lond. B Biol. Sci. 364, 399-407.

Liu, T., Larsson, J., and Carrasco, M. (2007). Feature-based attention modulates orientation-selective responses in human visual cortex. Neuron 55, 313-323.

Logothetis, N. K. (1998). Single units and conscious vision. Philos. Trans. R. Soc. Lond. B Biol. Sci. 353, 1801-1818.

Logothetis, N. K., Leopold, D., and Sheinberg, D. (1996). What is rivaling during binocular rivalry? Nature $380,621-624$.

Luck, S. J., Chelazzi, L., Hillyard, S. A., and Desimone, R. (1997). Neural mechanisms of spatial selective attention in areas $\mathrm{V} 1, \mathrm{~V} 2$, and $\mathrm{V} 4$ of macaque visual cortex. J. Neurophysiol. 77, 24-42.

Lumer, E., Friston, K., and Rees, G. (1998). Neural correlates of perceptual rivalry in the human brain. Science 280, 1930-1934.

McDougall, W. (1903). The physiological factors of the attention-process (III). Mind 12, 473-488.

McMains, S., and Kastner, S. (2011). Interactions of top-down and bottom-up mechanisms in human visual cortex. J. Neurosci. 31, 587-597.

Meng, M., and Tong, F. (2004). Can attention selectively bias bistable perception? Differences between binocular rivalry and ambiguous figures. J. Vis. 4, 539-551.

Mishra, J., and Hillyard, S. A. (2009). Endogenous attention selection during binocular rivalry at early stages of visual processing. Vision Res. 49, 1073-1080.

Mitchell, J., Stoner, G., and Reynolds, J. (2004). Object-based attention determines dominance in binocular rivalry. Nature 429, 410-413.

Moran, J., and Desimone, R. (1985). Selective attention gates visual processing in the extrastriate cortex. Science 229, 782-784.

Moray, N. (1970). Attention: Selective Processes in Vision and Hearing. New York: Academic Press.

Motter, B. C. (1993). Focal attention produces spatially selective processing in visual cortical areas V1, V2, and $\mathrm{V} 4$ in the presence of competing stimuli. J. Neurophysiol. 70, 909-919.

Mueller, T. J., and Blake, R. (1989). A fresh look at the temporal dynamics of binocular rivalry. Biol. Cybern. 61 , 223-232.

O'Connor, D. H., Fukui, M. M., Pinsk, M. A., and Kastner, S. (2002). Attention modulates responses in the human lateral geniculate nucleus. Nat. Neurosci. 5, 1203-1209.
Ooi, T. L., and He, Z. J. (1999). Binocular rivalry and visual awareness: the role of attention. Perception 28, 551-574.

Ooi, T. L., and He, Z. J. (2003). A distributed intercortical processing of binocular rivalry: psychophysical evidence. Perception 32, 155-166.

Paffen, C. L. E., and Alais, D. (2011). Attentional modulation of binocular rivalry. Front. Hum. Neurosci. 5:105. doi:10.3389/fnhum.2011.00105

Paffen, C. L. E., Alais, D., and Verstraten, F. A. J. (2006). Attention speeds binocular rivalry. Psychol. Sci. 17, 752-756.

Paffen, C. L. E., and Hooge, I. T. C. (2011). The effect of set size on the dynamics of binocular rivalry. Seeing Perceiving 24, 19-35.

Paffen, C. L. E., and van der Stigchel, S. (2010). Shifting spatial attention makes you flip: exogenous visual attention triggers perceptual alternations during binocular rivalry. Atten. Percept. Psychophys. 72, 1237-1243.

Paffen, C. L. E., Verstraten, F. A. J., and Vidnyánszky, Z. (2008). Attentionbased perceptual learning increases binocular rivalry suppression of irrelevant visual features. J. Vis. 8 , 25.21-11.

Pastukhov, A., and Braun, J. (2007). Perceptual reversals need no prompting by attention. J. Vis. 7, 5 .

Pearson, J., and Clifford, C. (2004). Determinants of visual awareness following interruptions during rivalry. J. Vis. 4, 196-202.

Pearson, J., Tadin, D., and Blake, R. (2007). The effects of transcranial magnetic stimulation on visual rivalry. J. Vis. 7, 2.1-11.

Peckham, R. H. (1936). Eye movements during "retinal rivalry." Am. J. Psychol. 48, 43-63.

Reynolds, J. H., and Chelazzi, L. (2004). Attentional modulation of visual processing. Annu. Rev. Neurosci. 27, 611-647.

Reynolds, J. H., Chelazzi, L., and Desimone, R. (1999). Competitive mechanisms subserve attention in macaque areas V2 and V4. J. Neurosci. 19, 1736-1753.

Roberts, M., Delicato, L. S., Herrero, J., Gieselmann, M. A., and Thiele, A. (2007). Attention alters spatial integration in macaque $\mathrm{V} 1$ in an eccentricity-dependent manner. Nat. Neurosci. 10 1483-1491.

Roeber, U. Veser, S., Schröger, E., and O'Shea, R. P. (2011). On the role of attention in binocular rivalry: electrophysiological evidence. PLoS ONE 6, e22612. doi:10.1371/journal.pone. 0022612
Serences, J. T., Schwarzbach, J., Courtney, S. M., Golay, X., and Yantis, S. (2004). Control of object-based attention in human cortex. Cereb. Cortex 14, 1346-1357.

Serences, J. T., and Yantis, S. (2006). Selective visual attention and perceptual coherence. Trends Cogn. Sci. (Regul. Ed.) 10, 38-45.

Shimojo, S., and Nakayama, K. (1990). Real world occlusion constraints and binocular-rivalry. Vision Res. 30, 69-80.

Silver, M. A., and Logothetis, N. K. (2004). Grouping and segmentation in binocular rivalry. Vision Res. 44, 1675-1692.

Silver, M. A., and Logothetis, N. K. (2007). Temporal frequency and contrast tagging bias the type of competition in interocular switch rivalry. Vision Res. 47, 532-543.

Simons, K. (2005). Amblyopia characterization, treatment, and prophylaxis. Surv. Ophthalmol. 50, 123-166.

Stanley, J., Carter, O., and Forte, J. (2011). Color and luminance influence, but can not explain, binocular rivalry onset bias. PLOS ONE 6, e18978. doi:10.1371/journal.pone.0018978

Stoner, G. R., Mitchell, J. F., Fallah, M., and Reynolds, J. H. (2005). Interacting competitive selection in attention and binocular rivalry. Prog. Brain Res. 149, 227-234.

Su, Y. R., He, Z. J., and Ooi, T. L. (2011). Revealing boundary-contour based surface representation through the time course of binocular rivalry. Vision Res. 51, 1288-1296.

Suzuki, S., and Grabowecky, M. (2007). Long-term speeding in perceptual switches mediated by attention-dependent plasticity in cortical visual processing. Neuron 56, 741-753.

Suzuki, S., and Peterson, M. A. (2000). Multiplicative effects of intention on the perception of bistable apparent motion. Psychol. Sci. 11, 202-209.

Tadin, D., Lappin, J. S., Blake, R., and Grossman, E. D. (2002). What constitutes an efficient reference frame for vision? Nat. Neurosci. 5, 1010-1015.

Tong, F., Meng, M., and Blake, R. (2006). Neural bases of binocular rivalry. Trends Cogn. Sci. (Regul. Ed.) 10, 502-511.

Toppino, T. C. (2003). Reversible-figure perception: mechanisms of intentional control. Percept. Psychophys. 65, 1285-1295.

Treue, S. (2001). Neural correlates of attention in primate visual cortex. Trends Neurosci. 24, 295-300. 
van Bogaert, E. A., Ooi, T. L., and He, Z. J. (2008). The monocularboundary-contour mechanism in binocular surface representation and suppression. Perception 37, 1197-1215.

van Dam, L. C. J., and van Ee, R. (2006). Retinal image shifts, but not eye movements per se, cause alternations in awareness during binocular rivalry. J. Vis. 6, 1172-1179.

van Ee, R., van Boxtel, J. J. A., Parker, A. L., and Alais, D. (2009). Multisensory congruency as a mechanism for attentional control over perceptual selection. J. Neurosci. 29, 11641-11649.

van Ee, R., van Dam, L., and Brouwer, G. (2005). Voluntary control and the dynamics of perceptual bi-stability. Vision Res. 45, 41-55.

Washburn, M. F., and Gillette, A. (1933). Studies from the psychological laboratory of Vassar College: LXII. Motor factors in voluntary control of cube perspective fluctuations and retinal rivalry fluctuations. Am. J. Psychol. 45, 315-319.

Wertheimer, M. (1938). "Laws of organization in perceptual forms," in $A$ Source Book of Gestalt Psychology, ed. W. D. Ellis (London: Harcourt Brace), 71-88.

Wilson, H. R. (2003). Computational evidence for a rivalry hierarchy in vision. Proc. Natl. Acad. Sci. U.S.A. 100, 14499-14503.

Wolfe, J. M. (1983). Influence of spatial frequency, luminance, and duration on binocular rivalry and abnormal fusion of briefly presented dichoptic stimuli. Perception 12, 447-456.

Xu, J. P., He, Z. J., and Ooi, T. L. (2010). Effectively reducing sensory eye dominance with a push-pull perceptual learning protocol. Curr. Biol. 20, 1864-1868.

$\mathrm{Xu}$, J. P., He, Z. J., and Ooi, T. L. (2011a). Perceptual learning to reduce sensory eye dominance beyond the focus of top-down visual attention. Vision Res. doi: 10.1016/j.visres.2011.05.013

$\mathrm{Xu}$, J. P., He, Z. J., and Ooi, T. L. (2011b). Push-pull training reduces foveal sensory dominance within the early visual channels. Vision Res. doi: 10.1016/j.visres.2011.06.005

Yang, E., Zald, D. H., and Blake, R. (2007). Fearful expressions gain preferential access to awareness during continuous flash suppression. Emotion 7 , 882-886.

Zaretskaya, N., Thielscher, A., Logothetis, N. K., and Bartels, A. (2010). Disrupting parietal function prolongs dominance durations in binocular rivalry. Curr. Biol. 20 2106-2111.

Zhang, P., Jamison, K., Engel, S., He, B. and $\mathrm{He}, \mathrm{S}$. (2011). Binocular rivalry requires visual attention. Neuron 71 , 362-369.
Conflict of Interest Statement: The authors declare that the research was conducted in the absence of any commercial or financial relationships that could be construed as a potential conflict of interest.

Received: 31 July 2011; paper pending published: 12 September 2011; accepted: 14 November 2011; published online: 02 December 2011.

Citation: Dieter KC and Tadin D (2011) Understanding attentional modulation of binocular rivalry: a framework based on biased competition. Front. Hum. Neurosci. 5:155. doi: 10.3389/fnhum.2011.00155

Copyright $(\odot 2011$ Dieter and Tadin. This is an open-access article distributed under the terms of the Creative Commons Attribution Non Commercial License, which permits non-commercial use, distribution, and reproduction in other forums, provided the original authors and source are credited. 